\title{
Calculation of the lifetimes of thin stripper targets under bombardment of intense pulsed ions
}

\author{
S. G. Lebedev ${ }^{1}$ and A. S. Lebedev ${ }^{2}$ \\ ${ }^{1}$ Institute for Nuclear Research of the Russian Academy of Sciences, 60th October Anniversary Prospect, 7a, Moscow, 117312, Russia \\ ${ }^{2}$ Lomonosov Moscow State University, Faculty of Calculus Mathematics and Cybernetics, Vorobievy Gory, GSP-2, Moscow, 119992, \\ Russia
}

(Received 10 June 2007; published 11 February 2008)

\begin{abstract}
The problems of stripper target behavior in the nonstationary intense particle beams are considered. The historical sketch on studies of radiation damage failure of carbon targets under ion bombardment is presented. The simple model of target evaporation under intensive pulsing beam is supposed. Lifetimes of stripper targets under intensive nonstationary beams can be described by two failure mechanisms: radiation damage accumulation and evaporation of a target. At the maximal temperatures less than $2500^{\circ} \mathrm{K}$ the radiation damage dominates; at temperatures above $2500^{\circ} \mathrm{K}$ the mechanism of evaporation of a foil prevails. The proposed approach has been applied to the description of stripper foils behavior in Brookhaven National Laboratory linac and Spallation Neutron Source conditions.
\end{abstract}

DOI: 10.1103/PhysRevSTAB.11.020401

PACS numbers: 29.27.- a, 81.40.Np, 85.70.Kh

\section{INTRODUCTION}

Thin solid-state targets are widely used in the world to strip ions in charged particle accelerators. The thickness of a stripper target is defined by the type, energy, and charge state of the ions before and after stripping. The processes occurring in a target bombarded by ion beams have been the subject of numerous studies over the past few decades. Extensive research in the mechanisms of destruction of targets by ion beams has been carried out in the 1970's and 1980 's - during a period of rapid development in particle accelerators and ion implantation techniques. With the recent development of high-intensity and high-energy ion accelerators at FermiLab and Spallation Neutron Source (SNS) in the U.S.A. and Japan Proton Accelerator Research Complex, the interest in studying the processes of failure of solid-state targets under ion beam bombardment has been renewed.

From the point of view of obtaining the highest charge states of a beam, loading of the vacuum system, and small overall dimensions, solid-state strippers are preferred compared to gaseous strippers. The main disadvantage of all solid-state strippers is their limited service life, which limits the overall performance of accelerators and raises the radiation load of service personnel.

From the first use of thin strippers until now, there has been a struggle to improve their manufacturing techniques with the purpose of increasing their lifetime. The wide use of carbon as a material for thin stripper targets is explained by its high melting point and mechanical strength. Because of its small nuclear mass carbon introduces minimal distortions to the parameters of a particle beam. In the late 1970's a prospective method of manufacturing carbon foils was developed - the cracking of hydrocarbon in glow discharge [1]. This method has been used for a long time for the manufacturing of long lifetime carbon foils. Later on, the method of manufacturing carbon foils by means of laser ablation was developed [2]. Longer lifetime foils were produced by this latter method. Recently, a significant improvement in the technology of making thin solid-state targets and increasing their lifetime took place at KEK by Isao Sugai and his group [3], who developed a whole series of methods of manufacturing of carbon targets. These methods are the controlled DC-arc discharge, the controlled AC-arc discharge, the mixed ionic beam sputtering, the high ion beam sputtering [3], and other methods.

\section{HISTORICAL SKETCH OF STUDYING THE BEHAVIOR OF CARBON TARGETS UNDER ION BOMBARDMENT}

The technique of quantitative estimations of the lifetime of carbon stripper targets was developed from the outset of their use. From behavior of an irradiated foil, it follows that the foil lifetime $t$ strongly depends on its crystal lattice destruction, therefore $t$ should be in inverse proportion to the displacement rate of target's atoms $K_{d}$. For the first time a semiempirical formula connecting $t$ and $K_{d}$ was presented in [4]. Yntema from Argonne National Laboratories (U.S.A.) and Nickel from GSI (Germany) have made a significant contribution to the development of the model of carbon films failure in ion beams [5]. For high-energy heavy ions the displacement rate can be expressed as follows:

$$
K_{d}=\frac{N_{0} \bar{\varphi}}{E_{d}} \int_{E_{d}}^{W_{\max }} W d \sigma(E, W),
$$

where $N_{0}$ is the full number of target atoms, $\bar{\varphi}$ is the flux density of bombarding particles averaged over a time (in particles $\left./ \mathrm{cm}^{2} / \mathrm{sec}\right), W$ is the recoil energy of the displaced atom of a target, $d \sigma$ is the differential cross section of scattering for single collision of an ion with an atom of the target, 


$$
W_{\max }=\frac{4 M_{1} M_{2} E}{\left(M_{1}+M_{2}\right)^{2}}
$$

is the maximal energy transferred in such a collision, $M_{1}$, $M_{2}$ is the masses of bombarding ion and target atom, respectively, $E$ is the energy of ion beam, and $E_{D}$ is the displacement energy of a target's atom. The basic assumption was that the target fails if half of its atoms are displaced from their initial positions [6]. Then the lifetime due to the destruction of a lattice can be estimated as

$$
t_{D}=\frac{E_{d} M_{2} E}{2 \pi \bar{\varphi} \mathrm{M}_{1} \mathrm{Z}_{1}^{2} \mathrm{Z}_{2}^{2} e^{4}}\left[\ln \left(\frac{W_{\max }}{E_{d}}\right)\right],
$$

where $Z_{1}$ and $Z_{2}$ are the charges of bombarding ion and target atom, respectively. The last expression was obtained using Rutherford's differential cross section, which is a good approximation at an ion specific energy above of $0.1 \mathrm{MeV} /$ nucleon. Equation (3) turned out to be wrong in the description of the experimental data due to neglecting the influence of target heating during irradiation. In the study of Yntema [7], it was shown that the heating of a foil increases its lifetime. This circumstance was taken into consideration in Ref. [6]. It was found to be possible to describe well the experimental results on the lifetimes of carbon foils by means of one semiempirical formula accounting for both the displacement of atoms of a target and heating:

$$
t=t_{D} a \exp \left(-\frac{b}{T}\right)
$$

where $a$ and $b$ are the empirical constants obtained by means of the least squares method from the experimental data, $T$ is the average temperature of a target defined by the Stephan-Boltzmann relation:

$$
T=\left(\frac{P}{2 \varepsilon \sigma}+T_{0}^{4}\right)^{1 / 4}, \quad P=\frac{d E}{d x} k h \bar{\varphi},
$$

where $d E / d x$ (in $\mathrm{MeV} \mathrm{cm}^{2} / \mathrm{g}$ ) is the electronic stopping power of the ion in a target material, $h$ the thickness of a target (in $\mathrm{g} / \mathrm{cm}^{2}$ ), $k=1.6 \times 10^{-13} \mathrm{~J} / \mathrm{MeV}$ the transfer factor, $T_{0}$ an ambient temperature, $\varepsilon$ the radiating ability of a foil, $\sigma=5.67 \times 10^{-12} \mathrm{~W} / \mathrm{cm}^{2} \mathrm{~K}^{4}$ StephanBoltzmann constant. The form of Eq. (4) allows one to use an analogy with the annealing of radiation defects with the activation energy $b$. The target lifetime increases with the increase of temperature, and, consequently, with the thickness increase which is in good agreement with numerous experiments. However, in Ref. [8] the experimental dependence (see Fig. 3 in [8]) of the lifetime decreasing with target thickness increasing is presented. However, the inverse relationship is caused by the reduction of transmission of ions with the increase of the thickness of a target above equilibrium, so the resulting dependence can be considered as atypical. The increase in the lifetime of a stripper foil with thickness increase occurs only at rather low temperatures $T<2500^{\circ} \mathrm{K}$ when there is no evaporation of a foil material. If evaporation takes place, the lifetime of a target, as will be seen below, decreases with the thickness increase. In [6] it is shown that, for thick enough targets $\left(h>10 \mu \mathrm{g} / \mathrm{cm}^{2}\right)$, the contribution of sputtering of atoms can be neglected in comparison with the radiation damage.

The stated technique has connected the destruction of carbon targets with the real processes in solid state-the creation of point defects under irradiation, and their migration and recombination. A disadvantage of the given technique is its empirical character. There is a natural desire to relate the empirical constants in formula (4) to real processes in a foil under irradiation. Though the meaning of a constant $b$ is intuitively clear, nevertheless it is necessary to tie up its value with a real value of the migration energy for the certain kind of point defects in real nanostructured carbon films. As for constant $a$, unfortunately it is not possible to relate its sense to the displacement of atoms of a lattice. A question arises: why does the failure of a film take place at the displacement of half of the atoms from their initial positions?

\section{INFLUENCE OF RADIATION DAMAGE ON THE FOIL LIFETIME}

One of the authors of this paper has devoted some years to the research of behavior of thin film targets under ion bombardment [9-13]. In Ref. [9] the relation described in (4) for the lifetime of a carbon foil has been deduced from the first principles of physics of radiation defects and the stress-deformed condition of solid state. It is known that under irradiation in the solid state two kinds of point radiation defects are created - the displaced atoms (or interstitials) and vacancies. The displaced atoms possess a high mobility. Most of them annihilate with vacancies in a lattice, and the rest form the molecular complexes. The sizes and number of the complexes depend on the temperature of irradiation, the defect generation rate, etc. The vacancies formed during irradiation usually remain isolated and inactive at low temperatures, but at high temperatures they also become mobile. As a result of irradiation, there is an accumulation of radiation defects, which deforms the crystal lattice. Around the vacancies there is a compression of the lattice. Because of the mobility of the displaced atoms a number of them recombine with the vacancies, and the rest create the complexes. As a result, a considerable amount of vacancies remain isolated and cause all-around compression of the crystal lattice. The deformation of the lattice causes internal pressure in the foil. If this pressure reaches the ultimate strength of a foil, it fails. This is a destruction picture of carbon foils under irradiation, which creates a physical basis for empirical expression (4). Omitting the detailed calculations, presented in [9-11], we shall write out the resulting relation for the lifetime: 


$$
t=0.23\left(\frac{3 \rho_{i} \sigma_{P}}{\xi \Delta \rho M}\right)^{3 / 2} \frac{\nu^{1 / 4}}{K_{d}^{5 / 4}} \exp \left(-\frac{E_{m}^{i}}{4 k_{B} T}\right) .
$$

Here, $\sigma_{P}$ is the ultimate strength, $M$ the elasticity module, $\nu$ the oscillation frequency of atoms in the lattice $(5 \times$ $\left.10^{13} \mathrm{~Hz}\right), E_{m}^{i}$ the migration energy of the displaced atoms in the foil, $k_{B}$ Boltzmann's constant, $\Delta \rho=\rho_{i}-\rho_{f}$ the change of density of a foil material due to irradiation, $\rho_{i}$ the density of the initial phase, and $\rho_{f}$ the density of the final phase. The factor $\xi$ defines the conditions of fastening a film on the frame (in case of rigid fastenings of a flat foil on the frame $\xi=1$ ).

For estimation of the rate of atom displacement it is possible to use the well-known expression,

$$
K_{d}=\frac{S_{n} \bar{\varphi}}{2 E_{D}}
$$

where $S_{n}$ characterizes the energy losses of a moving particle on the defects production. The expression for $S_{n}$, describing the experimental data on elastic scattering of ions on atoms, looks like this [14]:

$$
\begin{aligned}
S_{n}(E) & =\frac{4 \pi a Z_{1} Z_{2} e^{2} M_{1}}{M_{1}+M_{2}} S_{n}(\varepsilon), \\
S_{n}(\varepsilon) & =\frac{1.7 \sqrt{\varepsilon}(\ln \varepsilon+2.72)}{1+6.8 \varepsilon+3.4 \varepsilon^{3 / 2}}, \\
\varepsilon= & \frac{a M_{2} E}{Z_{1} Z_{2} e^{2}\left(M_{1}+M_{2}\right)}, \\
a & =\frac{0.9 a_{0}}{\left(\sqrt{Z_{1}}+\sqrt{Z_{2}}\right)^{2 / 3}} .
\end{aligned}
$$

In Eqs. (8) and (10) $e$ denotes the electron charge. The values of $M_{1}, M_{2}, Z_{1}$, and $Z_{2}$ are the same as used earlier in Eqs. (2) and (3). For carbon $E_{D}=25 \mathrm{eV}, a_{0}=0.53 \mathrm{~A}$. Expression (9) takes into account the screening of Coulomb's interactions at $\varepsilon \leq 10$. At $\varepsilon \gg 10$, by analogy with the work [6], it is possible to use Rutherford's expression for differential cross section:

$$
S_{n}=\frac{\ln \varepsilon}{2 \varepsilon} .
$$

The migration energy $E_{m}^{i}$ of the displaced atoms is related to the crystallite melting point $T_{m}$ by means of the expression

$$
E_{m}^{i}=k T_{m} .
$$

The crystallite size $L_{c}$ decreases its melting point according to Thomson's formula:

$$
T_{m}(L)=T_{m 0} \exp \left(-\frac{2 \sigma_{T}}{L_{c} \rho_{K} \Delta H_{0}}\right),
$$

where $T_{m 0}=4800^{\circ} \mathrm{K}, \Delta H_{0}=10 \mathrm{~kJ} / \mathrm{g}$ is the heat of fu- sion of infinitely long crystallite [15], $\rho_{K}=1.7 \mathrm{~g} / \mathrm{cm}^{3}$ is the average density of structure, and $\sigma_{T}=5.5 \times$ $10^{-4} \mathrm{~J} / \mathrm{cm}^{2}$ is the free surface energy of crystallite. The numerical values are presented for graphite. Then for the case of nanocrystalline graphite with the size $L_{c}=20 \mathrm{~A}$ that is characteristic for carbon foils obtained by cracking of ethylene in the glow discharge (GD-foils), the melting point will decrease to $3473^{\circ} \mathrm{K}$. Then for GD-foils the resulting formula for calculation of the lifetime can be written as follows [9]:

$$
t=50 K_{d}^{-(5 / 4)} \exp \left(-\frac{870}{T}\right) .
$$

The last expression has been used for the carbon stripper lifetimes and their comparison with the experimental data. The calculated results have been found to be in good agreement with the measured lifetimes for foil types GD and carbon arc $[9,10]$.

Expressions (6) and (15) for the lifetime of a carbon stripper foil under ion bombardment allow one to account for all important parameters: the temperature, the defect generation rate (or radiation damage) in a foil, the strength characteristics of a foil material, the migration energy of the displaced atoms and its dependence on the crystalline size, the conditions of fastening of a foil on the frame, and the oscillation frequency of atoms in a crystal lattice. The account of such a wide set of factors in the discussed expression for the lifetime opens opportunities for the description of irradiation behavior of carbon stripper targets obtained in various technological processes. The problem of calculation is that, in the experiments with a stripper target under ion bombardment, the measurements of the specified parameters are frequently not carried out.

The experience of using expression (15) for the prediction of a stripper target's behavior in the ion beams testifies to a satisfactory description of foil lifetime and its temperature dependence. Concerning displacement rate $K_{d}$, the expression (15) predicts nonlinear dependence $t \sim$ $\left(K_{d}\right)^{-1.25}$ which would be interesting to check experimentally. From expression (6) it is seen that $t \sim\left(\frac{\sigma_{P}}{M}\right)^{3 / 2}$ and this dependence also needs to be checked experimentally. The values of strength characteristics of carbon materials have a wide scatter depending on the manufacturing techniques. In work [16], the amorphous hydrocarbonic chemical vapor deposition (CVD) foils at a density of $2.19 \mathrm{~g} / \mathrm{cm}^{3}$ for the elastic modulus $\mathrm{M}$ have a value of $589 \mathrm{GPa}$ that makes about $52 \%$ of the elastic modulus of diamond. Hoshino et al. [17] reported that hydrogenated diamondlike carbon films, deposited in DC plasma of methane and hydrogen gas mixture at a particular anode and substrate position, had a high value of $850 \mathrm{GPa}$ for Young's modulus. This Young's modulus is about $74 \%$ of the directionally averaged value of diamond, $1141 \mathrm{GPa}$ [18]. In Ref. [19] for carbon nanotubes $M=1000 \mathrm{GPa}$, and the ultimate strength for nanotubes and diamond is $\sigma_{P}=100 \mathrm{Gpa}$; 
for graphite $M=10 \mathrm{GPa} \sigma_{P}=50 \mathrm{MPa}$ have been obtained. The limiting value of ultimate strength for CVD foils is $\sigma_{P}=47 \mathrm{MPa}$ [20]. The ultimate strength for GDfoils according to the authors of work [21] is $27 \mathrm{MPa}$. The authors of Ref. [22] for CVD-foils obtained the value $\sigma_{P}=$ $300 \mathrm{MPa}$. A film, obtained by the laser plasma ablation consisting of $75 \% s p^{3}$ and $25 \% s p^{2}$ coordinated atoms, has the elastic modulus $M=369 \mathrm{GPa}$ [23]. The decreasing quantity of a diamondlike phase gives rise to a decrease of the elastic modulus. The elastic modulus of foils, obtained by ion beam deposition, containing $0 \%-16 \%$ of $s p^{3}$-phase, varies within the limits from 100 up to $260 \mathrm{GPa}$ [24]. In all the presented experiments either the ultimate strength or the elastic modulus was measured; however, for the analysis of the lifetime of stripper targets, as follows from expression (6), it is necessary to know both values simultaneously. From the presented data it is seen that the strength characteristics of carbon materials strongly depend on the manufacturing techniques. For the diamond and nanotubes $\sigma_{P} \sim 0.1 \mathrm{M}$, that characterizes limiting value of strength. In the expression (15) for GD-foils the value $\sigma_{P} / M=0.01$ was used.

\section{EVAPORATION OF A TARGET BY AN INTENSE PULSING BEAM}

In the designing of intense accelerators of the charge particles, such as SNS, the estimation of the lifetime of stripper targets is important for definition of efficiency and radiation load on the personnel. Modeling the stripper targets behavior under irradiation at SNS was carried out at the Brookhaven National Laboratory (BNL) linac [25]. Parameters of a $H^{-}$beam at SNS and modeling beam at BNL are presented in Table I. For the analysis of the behavior of the SNS stripper target except for radiation damage, it is necessary to also consider the evaporation due to intensive heating by the circulating $\mathrm{H}^{+}$beam. The similar problem was solved in work [26] for stationary beams.

The energy losses of a bombarding beam in a target due to multiturn injection leads to heating up to $2500-4800^{\circ} \mathrm{K}$. This leads to sublimation of atoms from the surface of a target and its thickness decreases. On the other hand, with reduction of thickness, beam energy losses decrease. Therefore the temperature of the target decreases, the process of sublimation slows down, and the thickness of a target gets a new stationary value $h_{1}<h_{0}$. Thus, the processes of heating, sublimation, and change of thickness of a target are interdependent, and they must be taken into account in the description of evaporation of a target under irradiation.

The pressure of saturated vapor of carbon $P_{c}$ at the surface of a target essentially depends on its temperature. In a temperature range of $1700-5000^{\circ} \mathrm{K}$ this dependence can be presented in the form [27]

$$
P_{c}(T)=A \exp \left(-\frac{B}{T}\right)
$$

where $A=1.87 \times 10^{11}$ Torr, and $B=8.35 \times 10^{4} \mathrm{~K}$. The average rate of movement of carbon vapor atoms can be estimated as

$$
V=1.5 \times 10^{4} \sqrt{\frac{T}{M_{2}}}(\mathrm{~cm} / \mathrm{sec}),
$$

where $M_{2}$ is the atomic mass of carbon. The dependence of density of the carbon saturated vapor $n\left(\mathrm{~cm}^{3}\right)$ near the surface of target vs vapor pressure is given by the expression

$$
n=0.996 \times 10^{19} \frac{P_{c}[\text { Torr }]}{T[K]} .
$$

The quantity of atoms $N_{S}$ contained in $1 \mathrm{~cm}^{2}$ of the surface of a target with the thickness $h$ and density $\rho$, can be calculated as

$$
N_{S}=6 \times 10^{23} \frac{h \rho}{\mu_{c}},
$$

where $\mu_{c}=12 \mathrm{~g} /$ mole is the molar weight of carbon. The number of atoms leaving $1 \mathrm{~cm}^{2}$ of the surface of a target from its both sides is equal to $n V$. Then the variation rate of the quantity $N_{S}$ can be expressed as follows:

$$
\frac{d N_{S}}{d t}=-n V
$$

Differentiating the Eq. (19) with respect to time, we obtain

$$
\frac{d N_{s}}{d t}=6 \times 10^{23} \frac{\rho}{\mu_{c}} \frac{d h}{d t} .
$$

Substituting (21) in (20), and using (16)-(19), one can obtain the following differential equation, which allows one to calculate the dependence of thickness of a target $h$ vs time:

$$
\frac{d h(t)}{d t}=-8.12 \times 10^{10} \frac{[g \sqrt{K}]}{\left[\mathrm{cm}^{2} \mathrm{sec}\right]} \frac{\exp \left[\frac{-83500}{T}\right]}{\sqrt{T}} .
$$

TABLE I. The parameters of $H^{-}$beams at SNS and BNL linac according to Ref. [25].

\begin{tabular}{lccccc}
\hline \hline & Energy & Duration of an impulse & Frequency & The maximal current & The beam size \\
\hline SNS & $1 \mathrm{GeV}$ & $1 \mathrm{~ms}$ & $60 \mathrm{~Hz}$ & $32 \mathrm{~mA}$ & $3 \times 2 \mathrm{~mm}^{2}$ \\
BNL linac & $750 \mathrm{keV}$ & $0.5 \mathrm{~ms}$ & $6.7 \mathrm{~Hz}$ & $2.02 / 2.2 \mathrm{~mA}$ & $3 \mathrm{~mm} \mathrm{diameter}$ \\
\hline \hline
\end{tabular}


Unlike the case of stationary heating, considered in the work [26], the feature of loading of stripper targets at SNS, $\mathrm{BNL}$, and many other new facilities is a pulsing character of a bombarding beam. The average temperature of a target at $h_{0}=200 \mu \mathrm{g} / \mathrm{cm}^{2}$ makes only $997^{\circ} \mathrm{K}$; therefore the evaporation is improbable. However, calculations [25] show that at the same thickness the peak temperature in an impulse reaches $2350^{\circ} \mathrm{K}$. Therefore, in the analysis of experimental data on the behavior of a target at BNL linac conditions, it is important to consider the pulsing character of the $H^{-}$beam. The feature is that the evaporation occurs in that time interval of an impulse when the target is bombarded by ions, and the temperature of heating exceeds the threshold of sublimation.

For the adequate description of heating, cooling, and evaporation processes in a pulsing mode we use, similarly to the work [28], a nonstationary heat conduction equation. As a result, for the description of the interconnected processes of heating and evaporation of a foil in a pulsing beam of ions, we can obtain the following set of equations:

$$
\begin{gathered}
\frac{d T}{d t}=\frac{1}{C(T) h(t)}\left[P(t)+2 \varepsilon \sigma_{0} T_{0}^{4}-2 \varepsilon \sigma_{0} T^{4}(t)\right], \\
\frac{d h(t)}{d t}=-8.12 \times 10^{10} \frac{\exp \left[\frac{-83500}{T}\right]}{\sqrt{T}},
\end{gathered}
$$

where $P(t)$ is the pulse power, which is defined as

$$
P(t)=\varphi(t) \frac{d E}{d x} h_{0} k, \quad \frac{d E}{d x}=275 \frac{\mathrm{MeV} \mathrm{cm}^{2}}{\mathrm{~g}},
$$

$\varphi(t)$ is the pulse density of a flux of the bombarding particles, which is similar to the value of $\bar{\varphi}$, but defined through the pulse current, $T_{0}=293^{\circ} \mathrm{K}$ is the initial temperature of a film. In Eq. (23) the dependence of a thermal capacity $C$ vs temperature is used in the form of [28]:

$$
\begin{aligned}
C(T)= & 0.0127+2.872 \times 10^{-3} T-1.45 \times 10^{-6} T^{2} \\
& +3.12 \times 10^{-10} T^{3}-2.38 \times 10^{-14} T^{4} .
\end{aligned}
$$

\section{RESULTS OF CALCULATION AND DISCUSSION}

The solution of the set of Eqs. (23) and (24) was made by numerical methods by means of the program MATLAB7.0 (The MathWorks, Inc.). In Fig. 1 the calculation results of temperature of a BNL linac target are presented for the first second of irradiation at a pulse current of protons of $2 \mathrm{~mA}$. The contribution of stripped electrons to foil heating can be estimated as follows. Each $1 \mathrm{GeV}$ proton comes in with 2 electrons, and each electron has $545 \mathrm{keV}$ of kinetic energy. The total electron energy is about $1.6 \mathrm{~kW}$ which is substantially less compared to $2 \mathrm{MW}$ from protons. Note: only a small fraction of the electron energy is deposited into the foil.

The resulting maximal temperature is $2532^{\circ} \mathrm{K}$, which exceeds the value of $2350^{\circ} \mathrm{K}$, obtained for the similar case

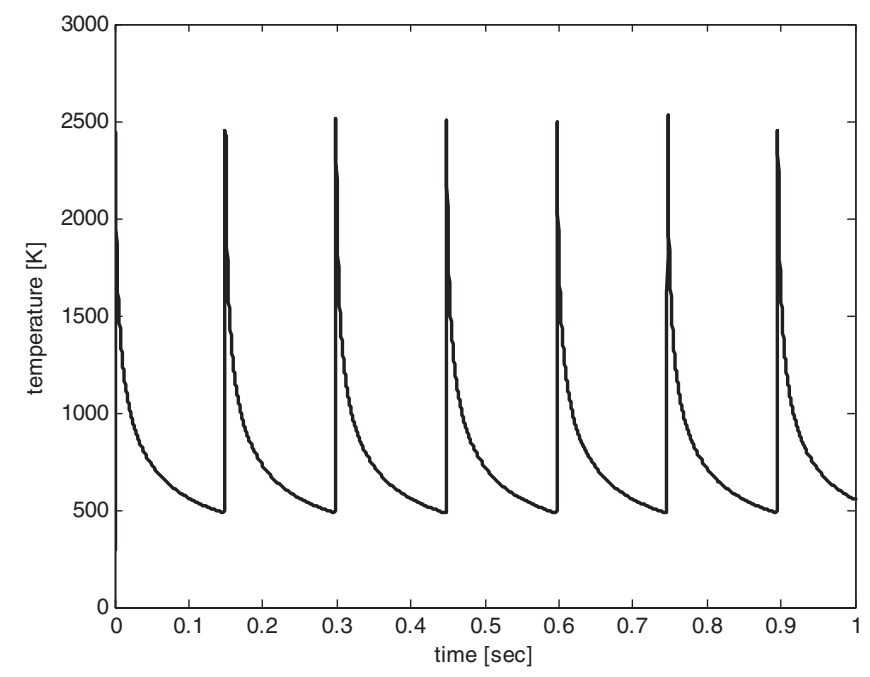

FIG. 1. A temperature field of a BNL linac target in the first second of work at a pulse current of $2 \mathrm{~mA}$.

in [25]. The distinction can be caused by using a temperature-dependent thermal capacity (26). The offered algorithm of calculation is powerful to account for the effect of the influence of heating and evaporation. In Figs. 2 and 3, the dependences of temperature and thickness of a foil vs irradiation time of a pulsing beam of BNL linac with a pulse current of $3 \mathrm{~mA}$ are shown. As it is possible to see, the evaporation rate and a temperature of a foil vary during the time. Such behavior can be explained in terms of interference of heating and evaporation. The results of calculation of a lifetime of a target are presented in Fig. 4 (please compare with Fig. 4 of [25]). For this calculation it was supposed that the lifetime corresponds to a reduction of the foil thickness by a half, though the given approximation demands further refinement. In the experi-

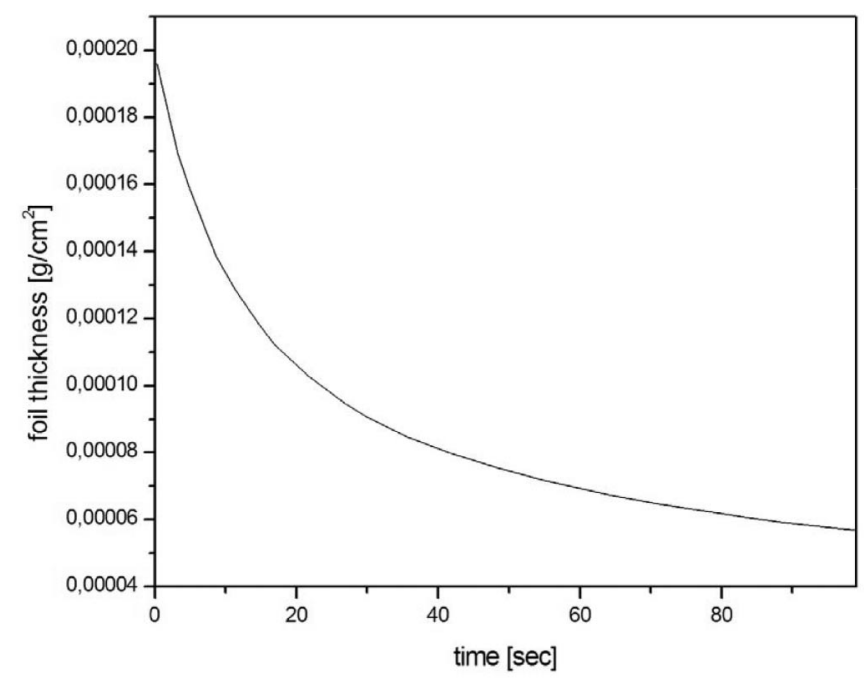

FIG. 2. Nonlinear foil thickness decreasing caused by the reduction of temperature at the BNL linac pulse current of $3 \mathrm{~mA}$. 


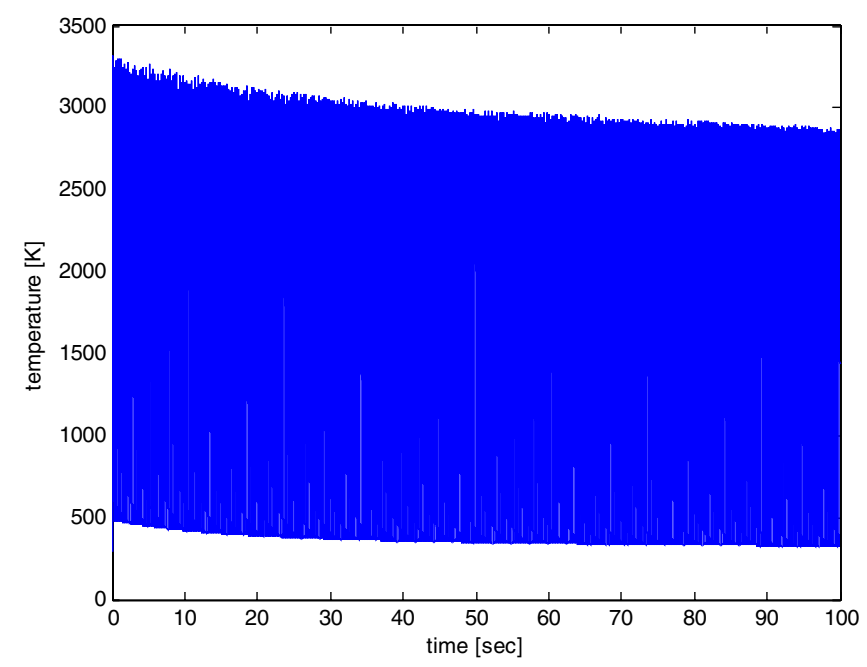

FIG. 3. (Color) Deformation of a temperature field in a target of BNL linac, caused by the decreasing of the foil thickness due to its evaporation.

ments [25], the target lifetime was defined as the time of reduction of the current through the foil by $10 \%$ There is a question: to what change in thickness does the given change in current correspond? For the answer it is necessary to measure the dependence of change in current behind the target vs change in its thickness. In Fig. 4 the results of calculation of the lifetime of a target at the BNL linac conditions, according to the mechanism of radiation damage are also presented. For the calculation of the temperature due to the mechanism of radiation damage, the relation (5) for a stationary heating was used. It is seen that for maximal temperatures less than $2500^{\circ} \mathrm{K}$ the radiation damage dominates; at temperatures above $2500^{\circ} \mathrm{K}$ the mechanism of foil evaporation prevails. As can be seen these two complementary pieces of dependence of foil lifetime vs maximum temperature well describes the experimental data of work [25]. In the case of foil evaporation, we use the universal critical parameters for saturated

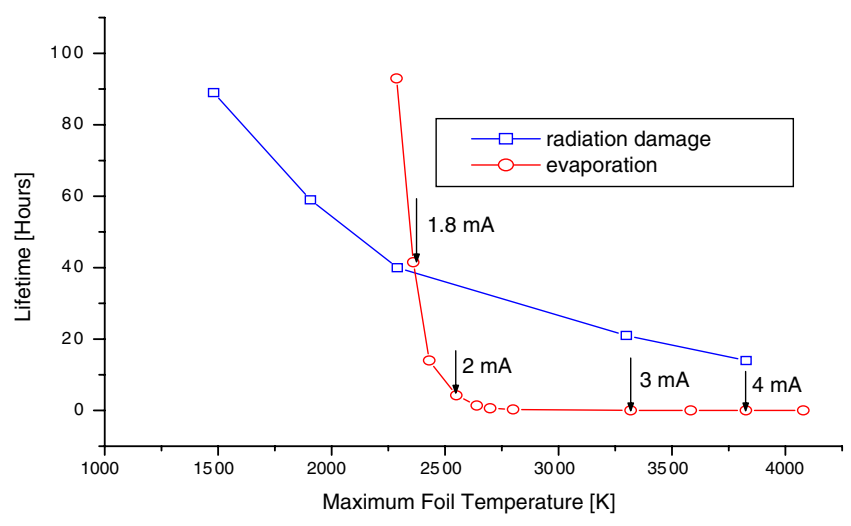

FIG. 4. (Color) Calculated dependences of the lifetime of BNL linac foil due to processes of radiation damage and evaporation. vapor of carbon from Ref. [27]. This means that in terms of evaporation there is no difference between various kinds of carbon materials such as graphite and diamond. So it is supposed that the lifetime of various kinds of carbons under evaporation is the same. As for the SNS stripper foil conditions presented in Table I, our calculation shows that the maximum foil temperature of $2650^{\circ} \mathrm{K}$ and the corresponding foil lifetime of $0.5-1$ hours can be obtained, which is limited by the evaporation process. As can be seen there is more than $30 \%$ decrease of the SNS stripper foil lifetime as compared with the modeling BNL linac case of Fig. 4, which can be attributed to the difference in the beam time structure. More exact evaluation of SNS stripper foil lifetime can be obtained by refining the differential equations (23) to account for the thermal conductivity and beam intensity distribution across the hot spot. A detailed computer simulation of the SNS foil temperature distribution is presented in Ref. [29].

\section{CONCLUSIONS}

(i) Lifetimes of stripper targets under intensive nonstationary beams can be described by two failure mechanisms: radiation damage accumulation and evaporation of a target. At the maximal temperatures less than $2500^{\circ} \mathrm{K}$ the radiation damage dominates; at temperatures above $2500^{\circ} \mathrm{K}$ the mechanism of evaporation of a foil prevails. (ii) The time structure of a pulsed beam can influence the lifetime. (iii) The strength characteristics of carbon material show considerable scattering. However, for the analysis of the lifetime of stripper targets, as follows from the expression (6), it is necessary to measure simultaneously both values: the elastic modulus $M$ and the ultimate strength $\sigma_{P}$. (iv) For a better description of stripper targets behavior in terms of radiation damage it is important to take into account the foil microstructure, the conditions of fastening of a film on the film frame, the change of density of a foil material due to irradiation. (v) It is necessary to carry out some experiments to check the dependence of the foil lifetime vs $K_{d}$ and $\sigma_{P}$.

[1] N. R. S. Tait, D. W. L. Tolfree, B.H. Armitage, and D. S. Whitmell, Nucl. Instrum. Methods 167, 21 (1979).

[2] P. Maier-Komor, G. Dollinger, and H. J. Korner, Nucl. Instrum. Methods Phys. Res., Sect. A 438, 73 (1999).

[3] I. Sugai, T. Hattori, H. Suzuki, H. Kinishita, H. Kato, and K. Yamazaki, Nucl. Instrum. Methods Phys. Res., Sect. A 265, 376 (1988).

[4] G. Frick, Rev. Phys. Appl. 12, 1525 (1977).

[5] J. Yntema and F. Nickel, Experimental Methods in Heavy Ion Physics, Lecture Notes in Physics Vol. 83 (SpringerVerlag, Berlin, 1978).

[6] F. Nickel, Nucl. Instrum. Methods 195, 457 (1982).

[7] J. Yntema, Nucl. Instrum. Methods 163, 1 (1979). 
[8] I. Sugai, Y. Takeda, M. Oyaizu, H. Kawakami, Y. Irie, K. Hara, A. Takagi, H. Hattori, K. Kawasaki, J. Kamiya, and M. Kinsho, Proceedings of HB2006, Tsukuba, Japan.

[9] E. A. Koptelov, S. G. Lebedev, and V. N. Panchenko, Nucl. Instrum. Methods Phys. Res., Sect. A A256, 247 (1987).

[10] E. A. Koptelov, S. G. Lebedev, and V. N. Panchenko, Nucl. Instrum. Methods Phys. Res., Sect. B 42, 239 (1989).

[11] S. G. Lebedev, Nucl. Instrum. Methods Phys. Res., Sect. B 85, 276 (1994).

[12] S. G. Lebedev, Nucl. Instrum. Methods Phys. Res., Sect. A 362, 160 (1995).

[13] S. G. Lebedev, Nucl. Instrum. Methods Phys. Res., Sect. A 397, 172 (1997).

[14] F. F. Komarov and M. A. Kumakhov, Radiat. Eff. 22, 1 (1974).

[15] V. N. Korobenko and A. I. Savvatimskiy, in Temperature: Its Measurement and Control in Science and Industry, edited by D.C. Ripple (American Institute of Physics Conference Proceedings, Melville, New York, 2003), Vol. 7, pp. 783-788.

[16] R. O. Dillon, Abbas Ali, N. J. Ianno, and A. Ahmad, J. Vac. Sci. Technol. A 19, 2826 (2001).

[17] S. Hoshino, K. Fujii, N. Shohata, H. Yamaguchi, Y. Tsukamoto, and M. Yanagisawa, J. Appl. Phys. 65, 1918 (1989).

[18] J.E. Field, in Diamond and Diamond-Like Films and Coatings, NATO-ASI-Series, edited by R.E. Clausing, L. L. Horton, J. C. Angus, and P. Koidl (Plenum, New
York, 1991), p. 17.

[19] Karl von Reden and Enid Sichel, in Proceedings of the Symposium of Northeastern Accelerator Personnel 2003, Strasbourg, FR.

[20] T. Call, J. O. Stoner, and S. Bashkin, Nucl. Instrum. Methods 167, 33 (1979).

[21] N. R. S. Tait, D. W. L. Tolfree, and B. H. Armitage, Nucl. Instrum. Methods 163, 1 (1979).

[22] D. V. Fedoseev, V. P. Varnin, and B. V. Deryagin, Fiz.-Him. Mech. Mater. 2, 118 (1977).

[23] F. Davanloo, T. J. Lee, D. R. Jander, H. Park, J. H. You, and C. B. Collins, J. Appl. Phys. 71, 1446 (1992).

[24] F. Rossi, B. Andre, A. Van Veen, P. E. Mijnarends, H. Schut, W. Gissler, J. Haupt, G. Lucazeau, and L. Abello, J. Appl. Phys. 75, 3121 (1994).

[25] C. J. Liaw, Y. Y. Lee, and J. Tuozzolo, Proceedings of Particle Accelerator Conference 2001, Chicago, IL, 2001, p. 1538

[26] B.P. Gikal, G. G. Gulbekyan, V.I. Kasacha, and D. V. Kamanin, Report No. JINR P9-2005-110, 2005.

[27] Ya. Groshkovsky, High-Vacuum Technique (Mir Publishing, Moscow, 1975).

[28] C. J. Liaw, Y. Y. Lee, J. Alessi, and J. Tuozzolo, in Proceedings Of Particle Accelerator Conference 1999, New York, p. 3300.

[29] J. Beebe-Wang, Y. Y. Lee, D. Raparia, and J.Wei, in Proceedings of Particle Accelerator Conference 2001, Chicago, IL, 2001, p. 1880. 